\title{
Time to Prepare for Risk Adaptation in Lymphoma by Standardizing Measurement of Metabolic Tumor Burden
}

\author{
Sally F. Barrington ${ }^{1}$ and Michel Meignan ${ }^{2}$ \\ ${ }^{1}$ Guy's and St. Thomas' PET Centre, School of Biomedical Engineering and Imaging Sciences, King's College London, King's Health \\ Partners, London, United Kingdom; and ${ }^{2}$ Lymphoma Study Association-Imaging (LYSA-IM), Functional Imaging and Therapeutics \\ Department, Henri Mondor University Hospitals, University Paris Est Créteil, Créteil, France
}

See an invited perspective on this article on page 1094.

Increased tumor burden is associated with inferior outcomes in many lymphoma subtypes. Surrogates of tumor burden that are easy to measure, such as the maximum tumor dimension of the bulkiest lesion on $\mathrm{CT}$, have been used as prognostic indices for many years. Recently, total metabolic tumor volume (MTV) and tumor lesion glycolysis have emerged as promising and robust biomarkers of outcome in various lymphomas. The median MTV and the optimal cutoffs to separate patients into risk groups in a study population are, however, highly dependent on the population characteristics and the delineation method used to outline tumor on the PET image. This issue has precluded the use of MTV for risk stratification in trials and clinical practice. Standardization of the methodology is timely to allow the potential for risk adaptation to be explored in addition to response adaptation using PET. Meetings between representatives from research groups active in the field were held under the auspices of the PET International Lymphoma and Myeloma Workshop. A summary of those discussions, which included a review of the literature and a practical assessment of methods used for outlining, including various software options, is presented. Finally, a proposal is made to perform a technical validation of MTV measurement enabling benchmark reference ranges to be derived for published delineation approaches used for outlining with various software. This process would require collation of representative imaging data sets of the most common lymphoma subtypes; agreement on pragmatic criteria for the selection of lesions; generation of a range of MTVs, with consensus to be reached on final contours in a training set; and development of automated software solutions with a set of minimum functionalities to reduce measurement variability. Methods developed in the above training exercise could then be applied to another data set, with a final set of contours and values generated. This final data set would provide a benchmark against which end-users could test their ability to measure MTVs that are consistent with expected values. The data set and automated software solutions could be shared with manufacturers with the aim of including these in standard workflows to allow standardization of MTV measurement across the world.

Key Words: lymphoma; positron emission tomography; standardization

J Nucl Med 2019; 60:1096-1102

DOI: 10.2967/jnumed.119.227249

Received Feb. 7, 2019; revision accepted Mar. 28, 2019.

For correspondence or reprints contact: Sally F. Barrington, School of Biomedical Engineering and Imaging Sciences, 4th Floor, Lambeth Wing, St. Thomas' Hospital, Westminster Bridge Rd., London SE1 7EH, U.K.

E-mail: sally.barrington@kcl.ac.uk

Published online Apr. 6, 2019.

COPYRIGHT (c) 2019 by the Society of Nuclear Medicine and Molecular Imaging.
$\mathbf{T}$ and inferior patient outcomes in Hodgkin lymphoma has been recognized since 1988 (1). At that time, tumor volume was assessed using clinical examination, chest radiography, and lymphography (1), later replaced by CT (2). These studies demonstrated that tumor burden was the single most important prognostic factor at the time of diagnosis for the prediction of treatment failure and disease relapse. The MabThera International Trial demonstrated the survival benefits of combining rituximab with chemotherapy in young patients with good-prognosis diffuse large B-cell lymphoma (3). In this landmark study, the presence of bulky disease was the only independent clinical risk factor associated with overall survival with a linear effect observed, using cutoffs from 6 to $10 \mathrm{~cm}$ for maximum tumor dimension. In a further trial in young patients with diffuse large B-cell lymphoma, with an age-adjusted International Prognostic Index of 1, a maximum tumor dimension of at least $10 \mathrm{~cm}$ was the only factor associated with overall survival (4). Similar findings were reported around the same time for follicular lymphoma, for which the longest diameter of the largest involved node was identified as an independent predictor of progression-free survival, with an optimal cutoff of $6 \mathrm{~cm} \mathrm{(5).}$

The time involved and the complexity of measuring the entire tumor volume in individual patients on CT scans have meant that surrogates for the total tumor burden have been relied on as predictive factors. Disease stage, number of involved nodes, involvement of extranodal sites, and the presence of bulk have been included in prognostic indices that are commonly used in Hodgkin and nonHodgkin lymphoma (5-9). These prognostic indices, however, are not very effective in classifying patients at high risk of treatment failure. Tumor volumes on PET and CT are routinely assessed for the purpose of radiotherapy planning, but this assessment is generally limited to one or a few sites rather than the total tumor burden.

The introduction of PET has made measurement of the total metabolically active volume of tumor more feasible. Tumor locations that accumulate ${ }^{18} \mathrm{~F}-\mathrm{FDG}$ can be outlined and summed to calculate the total metabolic tumor volume (MTV). Tumor lesion glycolysis (TLG) can also be assessed, which is the MTV multiplied by the $\mathrm{SUV}_{\text {mean }}$ in the entire volume and takes into account both the extent and the intensity of tracer uptake. Multiple reports from large studies performed on retrospective cohorts or retrospective analyses of prospective trials have demonstrated that MTV and/or TLG is associated with progression-free survival, and sometimes with overall survival, in subtypes including Hodgkin lymphoma, diffuse large B-cell lymphoma, follicular lymphoma, 
and primary mediastinal B-cell and T-cell lymphomas (10-18). Highly effective PET-adapted treatment may have contributed to the inability to show an association with overall survival in some studies. MTV appears to be a robust prognosticator irrespective of the method used for measurement. However, the median MTV or optimal cutoff that separates high- from lowrisk groups varies according to the patient population and the method of analysis. This variation has, to date, precluded the use of metabolic volumes for risk stratification in clinical trials on hematologic malignancies.

Standardization of the methodology for the assessment of metabolic tumor burden is required to validate this promising biomarker and enable inclusion in patient management. Standardization of response assessment with ${ }^{18} \mathrm{~F}$-FDG PET has previously been successful using the Deauville criteria (19), which are widely applied (20) and used for PET-response-adapted treatment (21). This standardization was undertaken as a sequential process. First, simple rules were agreed upon for reporting and measuring concordance rates among reviewers, using international cohorts of patients with Hodgkin lymphoma and non-Hodgkin lymphoma (technical validation) $(22,23)$. Second, the criteria were evaluated against patient outcomes in retrospective cohorts (24-27) and prospectively validated in clinical trials $(21,28,29)$. A similar approach to standardize the measurement of metabolic tumor burden is now proposed to enable testing of PET risk-adapted and response-adapted strategies.

Meetings were convened with representatives from research groups active in the field under the auspices of the PET International Lymphoma and Myeloma Workshop (https://www.lymphomapet. $\mathrm{com} /$ ). A review of the literature and studies in progress was undertaken, with presentations and face-to-face meetings in Paris on February 1, 2018, and Menton on October 4, 2018. A proposal was developed to perform a benchmarking exercise for the technical validation of MTV and TLG on ${ }^{18}$ F-FDG PET/CT images.

The group acknowledged uncertainties regarding which structures to include, which delineation methods to apply, and which software packages to use to outline tumor.

The following sections summarize the results of discussions and potential ways forward. The term cutoff is used to mean the MTV cutoff that separates patients into different risk groups. Threshold is used to mean the threshold applied in the segmentation method to delineate tumor.

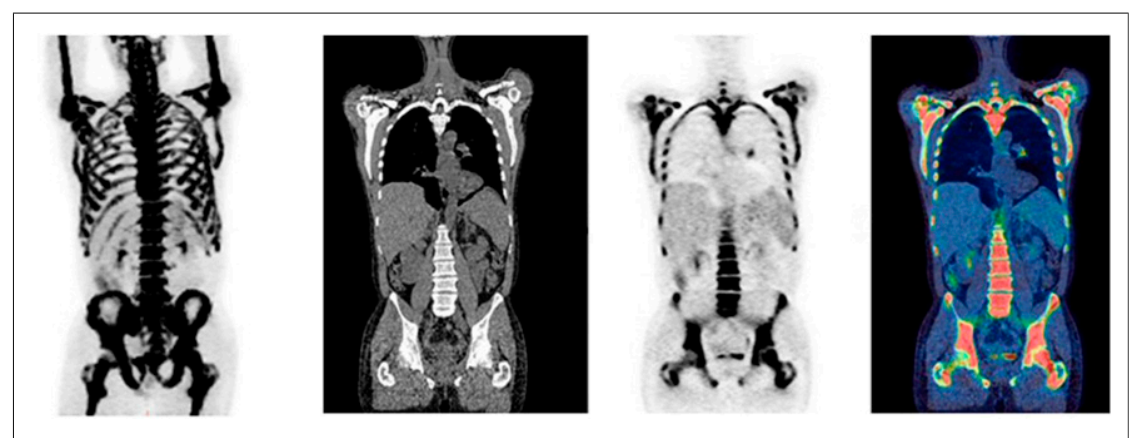

FIGURE 1. Maximum-intensity projection (left) CT, PET, and fused coronal images of patient with diffuse large B-cell lymphoma who had abnormally intense, diffuse uptake in bone marrow and minimal nodal involvement at left lung hilum. In this case, bone marrow involvement, which was confirmed on bone marrow biopsy, would seem appropriate to include in measurement of MTV.

\section{WHAT SHOULD BE INCLUDED IN THE ASSESSMENT OF MTV?}

Measurement begins with visual assessment of the scan, as occurs in routine clinical practice, noting the location of abnormal focal uptake in nodal and extranodal sites and ensuring that all relevant areas are imaged. Images should be scaled to a fixed SUV display and color table (20). Lymphomatous uptake can be distinguished from physiologic uptake and disease unrelated to lymphoma according to the distribution and CT characteristics with knowledge of the lymphoma subtype by a trained observer (20).

A pragmatic approach is required for measurement of MTV and TLG to be feasible in clinical practice, with the intention of capturing the main areas of tumor bulk. It may not be possible or desirable to include every small involved node or areas that are difficult to measure, such as diffuse disease in the bone marrow. A minimum volume, perhaps 2 or $3 \mathrm{~cm}^{3}$ at baseline, is suggested to avoid including multiple small regions that may be time-consuming to measure when a manual method is used but do not contribute much to the overall volume $(30,31)$. Smaller volumes may, however, need to be measured at the point of response assessment, as tumor residuals may be small. Acknowledging the uncertainties of this approach, technical validation could include measurement of the volume within compartments (e.g., nodal, splenic, and bone marrow compartments) as well as the total volume.

It is proposed to include, in the assessment of MTV and TLG, viable areas in lymph nodes with increased ${ }^{18} \mathrm{~F}$-FDG uptake above a specified threshold; focal uptake in the spleen, irrespective of splenic size; diffuse increased uptake in the spleen, in the absence of reactive changes in bone marrow, greater than the hepatic uptake (i.e., when there is a reversed hepatosplenic ratio); and focal uptake in the bone marrow. It is uncertain exactly how to classify an abnormal hepatosplenic ratio. Splenic uptake greater than 1.5 times the hepatic uptake has been used previously but has not been validated $(10,32,33)$. It is our experience that reactive changes in the bone marrow are often accompanied by similar changes in the spleen, and it is suggested that diffuse uptake in the spleen should not be included in the volume in this situation.

Diffuse uptake in the bone marrow occurs in approximately 1 in 5 patients with Hodgkin lymphoma (34) and is almost always due to reactive change; it was considered that such uptake should not be included in the MTV. In diffuse large B-cell lymphoma, diffuse uptake is more likely to indicate reactive change than lymphomatous involvement in the bone marrow compartment. However, when diffuse uptake is due to bone marrow involvement, the uptake usually reflects diffuse low-volume, and sometimes discordant, cellular infiltration (35-37), which probably has less of an impact on prognosis $(38,39)$ than areas of tumor bulk. In follicular lymphoma, diffuse cellular infiltration of the bone marrow is commonly missed by ${ }^{18}$ F-FDG PET (40). Patients with follicular lymphoma who are referred for PET scanning typically have a high tumor burden and are being considered for immunochemotherapy; for such patients, inclusion of bone marrow with diffuse cellular infiltration may be less important. For these reasons, it is suggested that in the computation of MTV, focal uptake be included only for the 3 


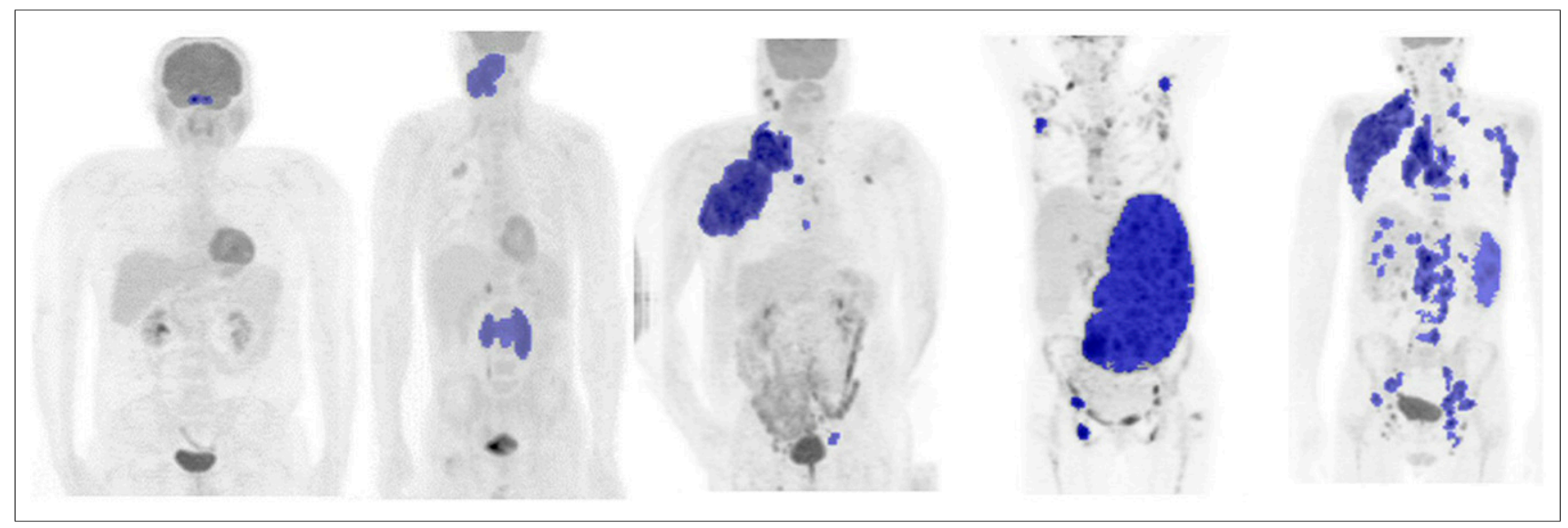

FIGURE 2. Examples of different sizes and distributions of tumor in 5 patients with diffuse large B-cell lymphoma.

most common lymphoma subtypes: Hodgkin lymphoma, diffuse large B-cell lymphoma, and follicular lymphoma. In occasional cases of diffuse large B-cell lymphoma, there may be mainly marrow-based disease, with abnormally intense, diffuse ${ }^{18} \mathrm{~F}-\mathrm{FDG}$ uptake confirmed on biopsy to represent bone marrow involvement; then, diffuse marrow uptake should be included in the measurement (Fig. 1).

\section{WHICH THRESHOLDS SHOULD BE APPLIED TO SEGMENT MTV?}

Satisfactory image quality and accurate quantification are key to ensuring reliable measurement of metabolic tumor burden. Solutions to deal with uncertainties in technical and biologic factors (41) are included in international guidance (42) and are commonly applied in trials and clinical practice for tumor imaging.

The segmentation of tumor is considerably more complex in patients with lymphoma than in those with solid tumors. There may be multiple sites of involvement in nodes and different extranodal sites, with large variability in lesion size and lesion shape, as well as heterogeneity of uptake and number (Fig. 2). Various contouring thresholds have been applied to outline tumor in lymphoma patients, perhaps because of this complexity. Results have been reported using absolute SUV thresholds applied to the entire image. The threshold may be fixed: for example, SUV equal to or greater than $2.5(13,16,43,44)$, SUV equal to or greater than 4.0 (45), or SUV relative to a reference region such as the liver or mediastinum $(46,47)$ as suggested in PERCIST (48). Results using percentage thresholds have also been reported: for example, outlining $41 \%(49-51)$ or $25 \%$ of the $\mathrm{SUV}_{\max }$ in individual lesions and then summing them to calculate MTV $(14,15)$. More complex image-processing methods, including gradient thresholds based on changes in the intensity of uptake at the edges of lesions (52); sourceto-background-corrected contours (53); and statistical methods such as clustering (54), fuzzy locally adaptive Bayesian (55), and others, have been proposed but not applied much in lymphoma and possibly have no clinical advantage over simpler methods (56).

The success of any delineation method will be influenced by tumor and imaging characteristics. The minimum SUV and $\mathrm{SUV}_{\text {max }}$ in the tumor and the spatial distribution will affect quantification $(53,57)$. Significant underestimation of visible tumor may occur with absolute thresholds if many voxels in a tumor mass have low uptake that is less than the threshold (Fig. 3), and conversely, overestimation of visible tumor may occur if tumor lies adjacent to areas of high physiologic uptake with spillover of counts into normal tissues (47). Underestimation occurs with percentage thresholds when there is a high SUV $\mathrm{max}_{\text {max }}$ and heterogeneity of uptake and many voxels have uptake that is lower than the threshold (Fig. 3); conversely, overestimation occurs when the $\mathrm{SUV}_{\max }$ in the tumor is low but significant (e.g., an $\mathrm{SUV}_{\max }$ of 4) and many voxels in the surrounding background are included in the contour.

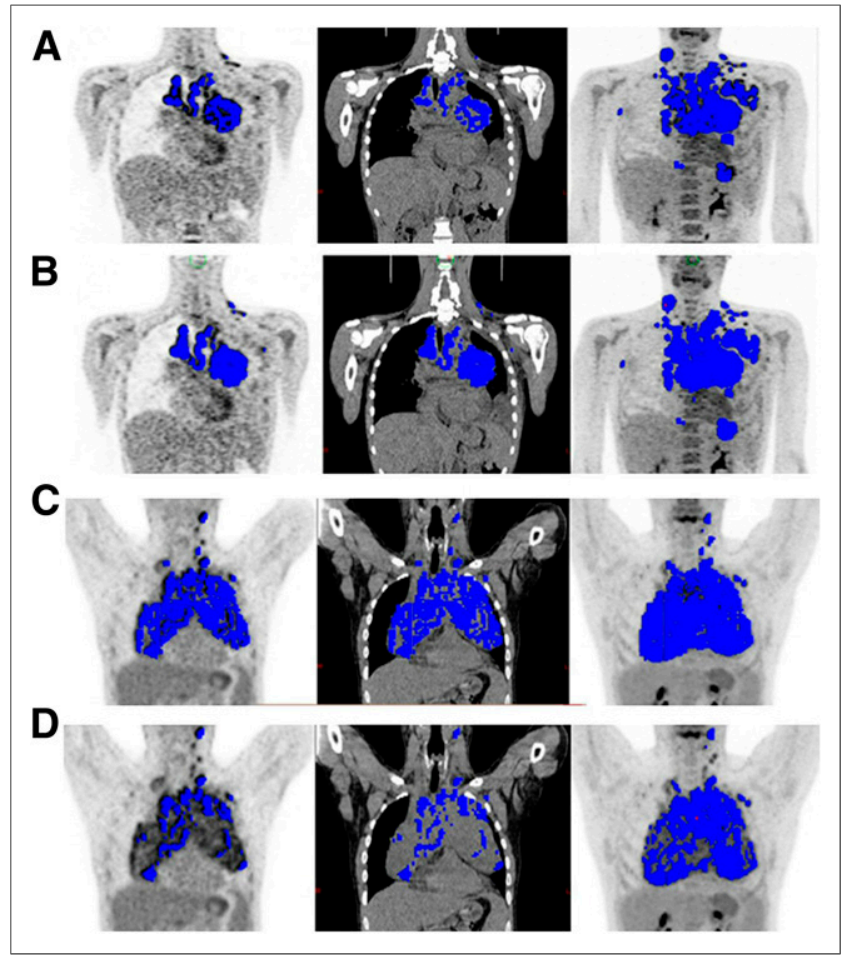

FIGURE 3. Representative PET (left), CT (middle), and maximum-intensity projection coronal images (right) for 2 patients. (A and B) Visible tumor is underestimated in one patient using percentage threshold $(A)$ compared with absolute threshold (B). (C and D) In another patient, visible tumor is adequately assessed using percentage threshold (C) but underestimated using absolute threshold (D). Same absolute threshold (SUV of 4$)$ and percentage threshold ( $41 \%$ of SUV $V_{\max }$ ) were used for both patients. 
Image noise, the matrix size, image resolution, and reconstruction will also affect SUVs $(58,59)$, although the impact of varying these parameters will be relatively more important in patients with smaller tumor volumes than in patients with advanced disease and large tumor volumes (53). MTV and TLG are much less sensitive to these influences than baseline metrics such as $\mathrm{SUV}_{\text {max }}$ and $\mathrm{SUV}_{\text {peak }}$, and MTV is less affected by these imaging characteristics than TLG (which is the product of MTV and the $\mathrm{SUV}_{\text {mean }}$ in the entire volume).

Irrespective of these challenges and the various thresholding methods applied to outline tumor in lymphoma, MTV and TLG remain strong prognostic indicators of patient outcomes (47). The different thresholds also appear to have good reproducibility between observers (47). Importantly, however, the use of different thresholds leads to different median values in study populations and, consequently, to different optimal cutoffs to separate patients into high- and low-risk groups (Table 1). The characteristics of the study population, including the range of volumes and the efficacy of treatment, also influence the cutoffs (60). The optimal cutoffs for prediction of risk using MTV and TLG may be unique to the particular patient characteristics, lymphoma subtype, and treatment and need to be derived for specific situations.

Each thresholding method clearly has limitations, and it currently may not be possible to decide on a single best method. It may be worthwhile to investigate approaches proposed in radiation oncology to reduce interobserver variation, whereby more than one threshold is combined using semiautomated contouring to outline tumors. These methods include the STAPLE algorithm (Simultaneous Truth and Performance Level Estimation) and the majority vote, for which only voxels selected using most segmentation methods are included in the final outline (61). Artificial intelligence methods also appear promising, with selection of imaging features used as the basis for choosing one of several segmentation methods in an individual patient (e.g., ATLAAS algorithm) (62). The rationale is that no single thresholding method will perform optimally in every patient but that the best-performing method in the majority of patients will be obtained by combining the voxels included in the tumor outline by more than one delineation method. Evaluation of absolute and percentage thresholds is likely to be required in a benchmarking exercise for the technical validation of MTV.

\section{WHICH SOFTWARE PACKAGES SHOULD BE USED, AND ARE MANUAL OR AUTOMATIC APPROACHES BETTER?}

Given that all thresholds appear to perform in a similar way to predict patient outcomes, the most important requirements for a suitable measurement method are high success rates for segmenting visible tumor, ease of use, and provision of quick, consistent results suitable for testing in multicenter trials and, ultimately, clinical application.

Various software options exist for measuring MTV and TLG, and some work better than others using different thresholds. Broadly speaking, most use some form of automatic segmentation that can then be adjusted manually. Such options may comprise the observer point-picking areas of tumor while avoiding areas of physiologic uptake or, in contrast, fully automated selection of regions of uptake applying one or more thresholds, with subsequent removal of physiologic uptake by the observer.

The former, using seed-growing algorithms for point-picking, is often easier when there are few areas of tumor present and they are well separated from areas of high uptake such as the brain, heart, or urinary system (Figs. 2C and 2D). In this scenario, the total MTV can be measured rapidly without the need for further editing, but the method is more observer-dependent and time-consuming than fully automatic segmentation when there is multifocal tumor.

Fully automated segmentation is easier with multiple tumor regions (Fig. 2E) but always requires removal of physiologic uptake. Cropping to avoid slices at the top (e.g., including brain uptake) and bottom (e.g., bladder uptake) of the image may reduce the amount of

TABLE 1

Characteristics Contributing to Different Medians and Optimal Cutoffs for Separating Patients into Risk Groups

\begin{tabular}{|c|c|c|c|c|c|c|c|c|c|c|c|}
\hline Study & $n$ & PFS and OS & $\geq 60 y$ & $\begin{array}{l}\text { Advanced } \\
\text { stage }\end{array}$ & Bulk & IPI & $\mathrm{PS} \geq 2$ & Threshold & $\begin{array}{c}\text { Median } \\
\left(\mathrm{cm}^{3}\right)\end{array}$ & $\begin{array}{c}\text { IQR } \\
\left(\mathrm{cm}^{3}\right)\end{array}$ & $\begin{array}{l}\text { Cutoff } \\
\left(\mathrm{cm}^{3}\right)\end{array}$ \\
\hline $\begin{array}{l}\text { Song } 2012 \\
(44)\end{array}$ & 169 & $\begin{array}{c}\text { 3-y PFS, 74; } \\
\text { OS, } 76\end{array}$ & $60 \%$ & $41 \%$ & $4 \% \geq 5 \mathrm{~cm}$ & $26 \% \geq 3$ & $25 \%$ & SUV $\geq 2.5$ & 198 & $5-1,991$ & 220 \\
\hline $\begin{array}{l}\text { Sasanelli } \\
2014(51)\end{array}$ & 114 & NA & $31 \%$ & $82 \%$ & $36 \% \geq 10 \mathrm{~cm}$ & $65 \% \geq 2^{*}$ & $30 \%$ & $\geq 41 \%$ SUV $_{\max }$ & 315 & $4-2,654$ & 550 \\
\hline $\begin{array}{l}\text { Song } 2016 \\
(43)\end{array}$ & 107 & NA & $67 \%$ & $100 \%$ & $19 \%$ & $81 \% \geq 4^{\dagger}$ & $16 \%$ & SUV $\geq 2.5$ & 527 & $15-3,549$ & 600 \\
\hline $\begin{array}{l}\text { Cottereau } \\
2016(49)\end{array}$ & 81 & $\begin{array}{c}\text { 5-y PFS, 60; } \\
\text { OS, } 63\end{array}$ & $63 \%$ & $80 \%$ & $40 \% \geq 10 \mathrm{~cm}$ & $68 \% \geq 2^{\star}$ & $30 \%$ & $\geq 41 \%$ SUV $_{\max }$ & 320 & $106-668$ & 300 \\
\hline $\begin{array}{l}\text { Mikhaeel } \\
2016 \text { (13) }\end{array}$ & 147 & $\begin{array}{c}\text { 5-y PFS, 65; } \\
\text { OS, } 74\end{array}$ & $48 \%$ & $69 \%$ & $40 \% \geq 10 \mathrm{~cm}$ & $69 \% \geq 2$ & $30 \%$ & SUV $\geq 2.5$ & 595 & $2-7,337$ & 400 \\
\hline Tout 2017 (66) & 108 & $\begin{array}{c}\text { 4-y PFS, 76; } \\
\text { OS, } 82\end{array}$ & $49 \pi$ & $80 \%$ & NA & $60 \% \geq 3^{\ddagger}$ & NA & $\geq 41 \%$ SUV $_{\max }$ & 313.5 & NA & NA \\
\hline
\end{tabular}

\footnotetext{
${ }^{*}$ Age-adjusted IPI.

${ }^{\dagger}$ National Comprehensive Cancer Network IPI.

${ }^{\ddagger}$ Modified IPI.

IMedian age.

PFS and OS data refer to percentages of patients.

$\mathrm{PFS}=$ progression-free survival; OS = overall survival; IPI = International Prognostic Index; $P S=$ performance status; IQR = interquartile range; $\mathrm{NA}=$ not available.
} 


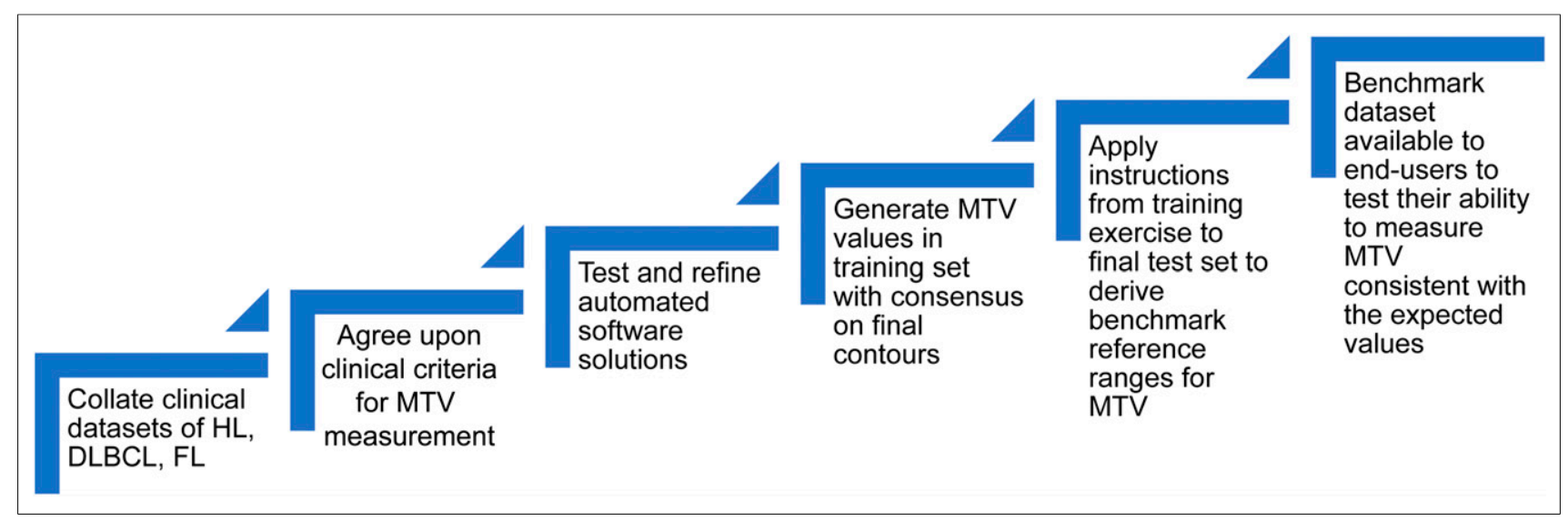

FIGURE 4. Road map for possible benchmarking exercise. DLBCL = diffuse large B-cell lymphoma; $F L=$ follicular lymphoma; $H L=H o d g k i n$ lymphoma.

editing required, if the tumor distribution allows, but this process is sometimes difficult, especially in follicular lymphoma.

The software that performs best will therefore vary by disease distribution and threshold chosen, and the 2 approaches should be combined in the same software package. Academic groups have developed shareware for research, recognizing that automation is highly desirable. These include LIFEx (https://www.lifexsoft.org) (63), FIJI (https://fiji.sc/), and ACCURATE (64). Ultimately, though, engagement with manufacturers is important for regulatory approval and for maintenance and development of the software for clinical use. Proprietary software solutions for measuring metabolic tumor burden using adaptive thresholding have been approved and, although useful for general reporting, are not widely applicable.

\section{WHERE SHOULD WE GO FROM HERE?}

It is proposed to collect representative baseline scans from patients with early and advanced Hodgkin lymphoma, early and advanced diffuse large B-cell lymphoma, and follicular lymphoma with high tumor burden. Scans could be collated from existing international published data sets (the number in each group to be decided) that are representative of the variation in ${ }^{18} \mathrm{~F}$-FDG uptake and image quality seen in clinical practice using a range of available technologies (Fig. 4).

Consensus criteria for inclusion of lesions in MTV and TLG could be formulated, on the basis of pragmatic choices, as suggested in this article. Measurement could be undertaken using available automated software developed by academic groups or, alternatively, using a new consensus method with region preselection based on the commonly applied absolute and percentage thresholds, with minimum volumes to be agreed upon based on similar work in radiation oncology (61). Using consensus criteria and automated selection of regions, MTVs and ranges could be generated for a training data set using 2 or more thresholds by observers from international groups. The final consensus contours should be agreed upon by an expert panel. Detailed instructions based on this training data set will allow reference MTVs to be generated for a separate test data set. This data set could provide a benchmark against which end-users in trials and clinical practice could test their ability to measure MTVs that are consistent with the expected values.

Automated software solutions could be shared with manufacturers, with a set of minimum functionalities required to minimize MTV measurement variability. Manufacturers should be encouraged to include these tools in standard work packages. This technical validation is the first step needed before MTV and TLG are prospectively or retrospectively tested as prognostic markers in specific populations to define cutoffs for risk stratification of patients treated with standard or experimental therapy. Risk stratification using MTV will likely involve integration with other baseline parameters such as clinical prognostic scores $(10,13,50)$, possibly as continuous variables and perhaps in combination with response assessment $(13,65)$.

\section{CONCLUSION}

We believe that segmentation of MTV should require minimal observer interaction (although this is inevitable in some cases); should not be vendor-specific; should work in different software environments; should require commercial support and regulatory approval; should be ideally integrated into the clinical workflow of all platforms, without the requirement to purchase separate packages for volume measurement; and should comply with the proposed benchmark standard as suggested in this paper. If these requirements are fulfilled, different software programs implementing the same delineation methods and used with the same settings should give MTVs within an acceptable prespecified range everywhere in the world.

\section{DISCLOSURE}

Sally Barrington acknowledges support from the National Institute for Health Research (RP-2-16-07-001). King's College London and UCL Comprehensive Cancer Imaging Centre are funded by the CRUK and EPSRC in association with the MRC and Department of Health and Social Care (England). Michel Meignan is the scientific coordinator of LYSA Imaging. LYSA is a cooperative group of hematologic centers involved in lymphoma care. It is a nonprofit organization supported by the French National Cancer Institute. The views expressed are those of the authors and not necessarily those of the NHS, the National Institute for Health Research, the Department of Health and Social Care (England) or LYSA. No other potential conflict of interest relevant to this article was reported.

\section{ACKNOWLEDGMENTS}

We thank our colleagues who contributed to this article, including the experts who participated in the focus group meeting 
in Paris (Ronald Boellaard, Irene Buvat, Olivier Casasnovas, Stephane Chauvie, Anne-Ségolene Cottereau, Andrea Gallamini, Salim Kanoun, Carsten Kobe, N. George Mikhaeel, Lucy Pike, Romain Ricci, and Annibale Versari), the experts who presented data or chaired sessions about this topic during the main PET International Lymphoma and Myeloma (PILM) Workshop in Menton (Ash Alizadeh, Ronald Boellaard, Irene Buvat, Olivier Casasnovas, Corinne Haioun, Martin Hutchings, Salim Kanoun, Lale Kostakoglu, David Kurtz, N. George Mikhaeel, Alison Moskowitz, Judith Trotman, Luigi Rigacci, Heiko Schöder, Lawrence Schwartz, Sigrid Stroobants, Hervé Tilly, and Jan Zaucha), and other members of the scientific committee who contributed to the closed expert session about this topic at PILM (Marc Andre, Abraham Avigdor, Stephanie Becker, Alberto Biggi, Francoise Bodéré, Caroline Bodet-Milin, Luca Ceriani, Monica Coronado, Eldad Dann, Ulrich Dührsen, Veronique Edeline, Tarec El-Galaly, Massimo Federico, Rosa Fonti, María José Garcia Velloso, Christian Gisselbrecht, Michele Gregianin, Corinne Haioun, Ken Herrmann, Andreas Hüttmann, Regina Klüge, Andrew Lister, Stefano Luminari, Maurizio Martelli, Philippe Moreau, Cristina Nanni, Wim Oyen, Marco Picardi, Alina Riedinger, Jesús San Miguel, Sigrid Stroobants, Ilan Tal, Jean-Noel Talbot, Catherine Thieblemont, Hervé Tilly, Emelie Van Zele, Thierry Vander Borght, Elena Zamagni, Josée Zijlstra, and Emanuele Zucca). We also thank Ronald Boellaard, Irene Buvat, Stephane Chauvie, Carsten Kobe, and Annibale Versari for reviewing and appraising the manuscript.

\section{REFERENCES}

1. Specht L, Nordentoft AM, Cold S, Clausen NT, Nissen NI. Tumor burden as the most important prognostic factor in early stage Hodgkin's disease: relations to other prognostic factors and implications for choice of treatment. Cancer. 1988; 61:1719-1727.

2. Gobbi PG, Ghirardelli ML, Solcia M, et al. Image-aided estimate of tumor burden in Hodgkin's disease: evidence of its primary prognostic importance. J Clin Oncol. 2001;19:1388-1394.

3. Pfreundschuh M, Ho AD, Cavallin-Stahl E, et al. Prognostic significance of maximum tumour (bulk) diameter in young patients with good-prognosis diffuse large-B-cell lymphoma treated with CHOP-like chemotherapy with or without rituximab: an exploratory analysis of the MabThera international trial group (MInT) study. Lancet Oncol. 2008;9:435-444.

4. Récher C, Coiffier B, Haioun C, et al. Intensified chemotherapy with ACVBP plus rituximab versus standard $\mathrm{CHOP}$ plus rituximab for the treatment of diffuse large B-cell lymphoma (LNH03-2B): an open-label randomised phase 3 trial. Lancet. 2011;378:1858-1867.

5. Federico M, Bellei M, Marcheselli L, et al. Follicular lymphoma international prognostic index 2: a new prognostic index for follicular lymphoma developed by the international follicular lymphoma prognostic factor project. J Clin Oncol. 2009;27:4555-4562.

6. Hasenclever D, Diehl V. A prognostic score for advanced Hodgkin's disease: international prognostic factors project on advanced Hodgkin's disease. $N$ Engl J Med. 1998;339:1506-1514.

7. Solal-Céligny P, Roy P, Colombat $\mathrm{P}$, et al. Follicular lymphoma international prognostic index. Blood. 2004;104:1258-1265.

8. Zhou Z, Sehn LH, Rademaker AW, et al. An enhanced international prognostic index (NCCN-IPI) for patients with diffuse large B-cell lymphoma treated in the rituximab era. Blood. 2014;123:837-842.

9. International Non-Hodgkin's Lymphoma Prognostic Factors Project: a predictive model for aggressive non-Hodgkin's lymphoma. N Engl J Med. 1993;329:987994.

10. Meignan M, Cottereau AS, Versari A, et al. Baseline metabolic tumor volume predicts outcome in high-tumor-burden follicular lymphoma: a pooled analysis of three multicenter studies. J Clin Oncol. 2016;34:3618-3626.

11. Cottereau AS, El-Galaly TC, Becker S, et al. Predictive value of PET response combined with baseline metabolic tumor volume in peripheral T-cell lymphoma patients. J Nucl Med. 2018;59:589-595.

12. Cottereau AS, Versari A, Loft A, et al. Prognostic value of baseline metabolic tumor volume in early-stage Hodgkin lymphoma in the standard arm of the H10 trial. Blood. 2018;131:1456-1463.
13. Mikhaeel NG, Smith D, Dunn JT, et al. Combination of baseline metabolic tumour volume and early response on PET/CT improves progression-free survival prediction in DLBCL. Eur J Nucl Med Mol Imaging. 2016;43:1209-1219.

14. Ceriani L, Martelli M, Zinzani PL, et al. Utility of baseline ${ }^{18}$ FDG-PET/CT functional parameters in defining prognosis of primary mediastinal (thymic) large B-cell lymphoma. Blood. 2015;126:950-956.

15. Ceriani L, Milan L, Martelli M, et al. Metabolic heterogeneity on baseline ${ }^{18}$ FDG$\mathrm{PET} / \mathrm{CT}$ scan is a predictor of outcome in primary mediastinal B-cell lymphoma. Blood. 2018;132:179-186.

16. Akhtari M, Milgrom SA, Pinnix CC, et al. Reclassifying patients with earlystage Hodgkin lymphoma based on functional radiographic markers at presentation. Blood. 2018;131:84-94.

17. Pike LC, Kirkwood AA, Patrick P, et al. Can baseline PET-CT features predict outcomes in advanced Hodgkin lymphoma? A prospective evaluation of UK patients in the RATHL trial (CRUK/07/033). Hematol Oncol. 2017;35:37-38.

18. Moskowitz AJ, Schoder H, Gavane S, et al. Prognostic significance of baseline metabolic tumor volume in relapsed and refractory Hodgkin lymphoma. Blood. 2017;130:2196-2203.

19. Meignan M, Gallamini A, Meignan M, Gallamini A, Haioun C. Report on the first international workshop on interim-PET-scan in lymphoma. Leuk Lymphoma. 2009;50:1257-1260.

20. Barrington SF, Mikhaeel NG, Kostakoglu L, et al. Role of imaging in the staging and response assessment of lymphoma: consensus of the International Conference on Malignant Lymphomas Imaging Working Group. J Clin Oncol. 2014;32:3048-3058.

21. Johnson P, Federico M, Kirkwood A, et al. Adapted treatment guided by interim PETCT scan in advanced Hodgkin's lymphoma. N Engl J Med. 2016;374:2419-2429.

22. Itti E, Meignan M, Berriolo-Riedinger A, et al. An international confirmatory study of the prognostic value of early PET/CT in diffuse large B-cell lymphoma: comparison between Deauville criteria and SSUVmax. Eur J Nucl Med Mol Imaging. 2013;40:1312-1320.

23. Biggi $A$, Gallamini $A$, Chauvie $S$, et al. International validation study for interim PET in ABVD-treated, advanced-stage Hodgkin lymphoma: interpretation criteria and concordance rate among reviewers. J Nucl Med. 2013;54:683-690.

24. Gallamini A, Barrington SF, Biggi A, et al. The predictive role of interim positron emission tomography for Hodgkin lymphoma treatment outcome is confirmed using the interpretation criteria of the Deauville five-point scale. Haematologica. 2014;99:1107-1113.

25. Trotman J, Luminari S, Boussetta S, et al. Prognostic value of PET-CT after firstline therapy in patients with follicular lymphoma: a pooled analysis of central scan review in three multicentre studies. Lancet Haematol. 2014;1:e17-e27.

26. Nols N, Mounier N, Bouazza S, et al. Quantitative and qualitative analysis of metabolic response at interim positron emission tomography scan combined with international prognostic index is highly predictive of outcome in diffuse large B-cell lymphoma. Leuk Lymphoma. 2014;55:773-780.

27. Kobe C, Goergen H, Baues C, et al. Outcome-based interpretation of early interim PET in advanced-stage Hodgkin lymphoma. Blood. 2018;132:2273-2279.

28. Trotman J, Barrington SF, Belada D, et al. Prognostic value of end-of-induction PET response after first-line immunochemotherapy for follicular lymphoma (GALLIUM): secondary analysis of a randomised, phase 3 trial. Lancet Oncol. 2018;19:1530-1542.

29. Mamot C, Klingbiel D, Hitz F, et al. Final results of a prospective evaluation of the predictive value of interim positron emission tomography in patients with diffuse large B-cell lymphoma treated with R-CHOP-14 (SAKK 38/07). J Clin Oncol. 2015;33:2523-2529.

30. Daisne JF, Sibomana M, Bol A, Doumont T, Lonneux M, Gregoire V. Tridimensional automatic segmentation of PET volumes based on measured source-tobackground ratios: influence of reconstruction algorithms. Radiother Oncol. 2003; 69:247-250.

31. Tylski P, Stute S, Grotus N, et al. Comparative assessment of methods for estimating tumor volume and standardized uptake value in ${ }^{18} \mathrm{~F}$-FDG PET. $\mathrm{J}$ Nucl Med. 2010;51:268-276.

32. Meignan M, Sasanelli M, Casasnovas RO, et al. Metabolic tumour volumes measured at staging in lymphoma: methodological evaluation on phantom experiments and patients. Eur J Nucl Med Mol Imaging. 2014;41:1113-1122.

33. Casasnovas O, Collin A, Kanoun S, et al. Spleen involvement identified on baseline PET imaging influences outcome of young patients with high risk diffuse large B-cell lymphoma treated with R-CHOP14 but not R-ACVBP [abstract]. Hematol Oncol. 2015;33(suppl):211.

34. Voltin CA, Goergen H, Baues C, et al. Value of bone marrow biopsy in Hodgkin lymphoma patients staged by FDG PET: results from the German Hodgkin study group trials HD16, HD17, and HD18. Ann Oncol. 2018;29:1926-1931.

35. Cerci JJ, Gyorke T, Fanti S, et al. Combined PET and biopsy evidence of marrow involvement improves prognostic prediction in diffuse large B-cell lymphoma. J Nucl Med. 2014;55:1591-1597. 
36. Alzahrani M, El-Galaly TC, Hutchings M, et al. The value of routine bone marrow biopsy in patients with diffuse large B-cell lymphoma staged with PET/CT: a Danish-Canadian study. Ann Oncol. 2016;27:1095-1099.

37. Khan AB, Barrington SF, Mikhaeel NG, et al. PET-CT staging of DLBCL accurately identifies and provides new insight into the clinical significance of bone marrow involvement. Blood. 2013;122:61-67.

38. Campbell J, Seymour JF, Matthews J, Wolf M, Stone J, Juneja S. The prognostic impact of bone marrow involvement in patients with diffuse large cell lymphoma varies according to the degree of infiltration and presence of discordant marrow involvement. Eur J Haematol. 2006;76:473-480.

39. Sehn LH, Scott DW, Chhanabhai M, et al. Impact of concordant and discordant bone marrow involvement on outcome in diffuse large B-cell lymphoma treated with R-CHOP. J Clin Oncol. 2011;29:1452-1457.

40. Luminari S, Biasoli I, Arcaini L, et al. The use of FDG-PET in the initial staging of 142 patients with follicular lymphoma: a retrospective study from the FOLL05 randomized trial of the Fondazione Italiana Linfomi. Ann Oncol. 2013;24:21082112 .

41. Boellaard R. Standards for PET image acquisition and quantitative data analysis. J Nucl Med. 2009;50(suppl 1):11S-20S.

42. Boellaard R, Delgado-Bolton R, Oyen WJ, et al. FDG PET/CT: EANM procedure guidelines for tumour imaging: version 2.0. Eur J Nucl Med Mol Imaging. 2015;42:328-354.

43. Song MK, Yang DH, Lee GW, et al. High total metabolic tumor volume in PET/ CT predicts worse prognosis in diffuse large B cell lymphoma patients with bone marrow involvement in rituximab era. Leuk Res. 2016;42:1-6.

44. Song MK, Chung JS, Shin HJ, et al. Clinical significance of metabolic tumor volume by PET/CT in stages II and III of diffuse large B cell lymphoma without extranodal site involvement. Ann Hematol. 2012;91:697-703.

45. Kurtz DM, Green MR, Bratman SV, et al. Noninvasive monitoring of diffuse large B-cell lymphoma by immunoglobulin high-throughput sequencing. Blood. 2015;125:3679-3687.

46. Kostakoglu L, Martelli M, Sehn LH, et al. Baseline PET-derived metabolic tumor volume metrics predict progression-free and overall survival in DLBCL after first-line treatment: results from the phase 3 GOYA study [abstract]. Blood. 2017;130(suppl 1):824.

47. Ilyas H, Mikhaeel NG, Dunn JT, et al. Defining the optimal method for measuring baseline metabolic tumour volume in diffuse large B cell lymphoma. Eur J Nucl Med Mol Imaging. 2018;45:1142-1154.

48. Wahl RL, Jacene H, Kasamon Y, Lodge MA. From RECIST to PERCIST: evolving considerations for PET response criteria in solid tumors. J Nucl Med. 2009;50(suppl 1):122S-150S.

49. Cottereau AS, Lanic H, Mareschal S, et al. Molecular profile and FDG-PET/CT total metabolic tumor volume improve risk classification at diagnosis for patients with diffuse large B-cell lymphoma. Clin Cancer Res. 2016;22:3801-3809.

50. Cottereau AS, Becker S, Broussais F, et al. Prognostic value of baseline total metabolic tumor volume (TMTV0) measured on FDG-PET/CT in patients with peripheral T-cell lymphoma (PTCL). Ann Oncol. 2016;27:719-724.
51. Sasanelli M, Meignan M, Haioun C, et al. Pretherapy metabolic tumour volume is an independent predictor of outcome in patients with diffuse large B-cell lymphoma. Eur J Nucl Med Mol Imaging. 2014;41:2017-2022.

52. Geets X, Lee JA, Bol A, Lonneux M, Gregoire V. A gradient-based method for segmenting FDG-PET images: methodology and validation. Eur J Nucl Med Mol Imaging. 2007;34:1427-1438.

53. Nestle U, Kremp S, Schaefer-Schuler A, et al. Comparison of different methods for delineation of ${ }^{18} \mathrm{~F}$-FDG PET-positive tissue for target volume definition in radiotherapy of patients with non-small cell lung cancer. J Nucl Med. 2005;46: 1342-1348.

54. Belhassen S, Zaidi H. A novel fuzzy C-means algorithm for unsupervised heterogeneous tumor quantification in PET. Med Phys. 2010;37:1309-1324.

55. Hatt M, Laurent B, Fayad H, Jaouen V, Visvikis D, Le Rest CC. Tumour functional sphericity from PET images: prognostic value in NSCLC and impact of delineation method. Eur J Nucl Med Mol Imaging. 2018;45:630-641.

56. Cottereau AS, Hapdey S, Chartier L, et al. Baseline total metabolic tumor volume measured with fixed or different adaptive thresholding methods equally predicts outcome in peripheral T cell lymphoma. J Nucl Med. 2017;58:276-281.

57. Boellaard R, Krak NC, Hoekstra OS, Lammertsma AA. Effects of noise, image resolution, and ROI definition on the accuracy of standard uptake values: a simulation study. J Nucl Med. 2004;45:1519-1527.

58. Kuhnert G, Boellaard R, Sterzer S, et al. Impact of PET/CT image reconstruction methods and liver uptake normalization strategies on quantitative image analysis. Eur J Nucl Med Mol Imaging. 2016;43:249-258.

59. Krak NC, Boellaard R, Hoekstra OS, Twisk JW, Hoekstra CJ, Lammertsma AA. Effects of ROI definition and reconstruction method on quantitative outcome and applicability in a response monitoring trial. Eur J Nucl Med Mol Imaging. 2005;32: 294-301.

60. Schöder H, Moskowitz C. Metabolic tumor volume in lymphoma: hype or hope? J Clin Oncol. 2016;34:3591-3594.

61. Schaefer A, Vermandel M, Baillet C, et al. Impact of consensus contours from multiple PET segmentation methods on the accuracy of functional volume delineation. Eur J Nucl Med Mol Imaging. 2016;43:911-924.

62. Berthon B, Marshall C, Evans M, Spezi E. ATLAAS: an automatic decision treebased learning algorithm for advanced image segmentation in positron emission tomography. Phys Med Biol. 2016;61:4855-4869.

63. Nioche C, Orlhac F, Boughdad S, et al. LIFEx: a freeware for radiomic feature calculation in multimodality imaging to accelerate advances in the characterization of tumor heterogeneity. Cancer Res. 2018;78:4786-4789.

64. Boellaard R. Quantitative oncology molecular analysis suite: ACCURATE [abstract]. J Nucl Med. 2018;59(suppl 1):1753.

65. Cottereau AS, Versari A, Luminari S, et al. Prognostic model for high-tumorburden follicular lymphoma integrating baseline and end-induction PET: a LYSA/ FIL study. Blood. 2018;131:2449-2453.

66. Tout M, Casasnovas O, Meignan M, et al. Rituximab exposure is influenced by baseline metabolic tumor volume and predicts outcome of DLBCL patients: a lymphoma study association report. Blood. 2017;129:2616-2623. 